\title{
Evaluation of a Pharmacist-Led Intervention to Improve Statin Use in Persons with Diabetes
}

\author{
Sarah L. Anderson, PharmD; Joel C. Marrs, PharmD, MPH; Cynthia R. Chachas, PharmD; \\ Brian S. Cichon, MPH; Amber D. Cizmic, PharmD; Bianca B. Calderon, PharmD; \\ and Tara B. Vlasimsky, PharmD
}

\begin{abstract}
BACKGROUND: The Statin Use in Persons with Diabetes (SUPD) measure, developed and endorsed by the Pharmacy Quality Alliance and National Quality Forum, has been adopted by the Centers for Medicare \& Medicaid Services as part of the star rating measure set. It was added as a display measure in 2015 and, as of 2019, has become a star measure. Clinical pharmacy specialists (CPS) embedded in the patient-centered medical home (PCMH) are well positioned to review and recommend statin therapy for patients with diabetes in order to improve patient care and health plan performance.
\end{abstract}

OBJECTIVE: To improve rates of statin prescribing and performance on the SUPD measure in the Denver Health Medical Plan (DHMP) population with diabetes by creating a CPS-led intervention to initiate statin prescriptions in eligible patients.

METHODS: Between February 1, 2018, and December 31, 2018, DHMP patients who met SUPD measure criteria (aged $\geq 40$ and $\leq 75$ years, dispensing events for at least 2 diabetes mellitus medication fills, and no statin prescribed) were identified by the health plan chart review and contacted by CPS as appropriate. For patients eligible and agreeable to statin therapy, the CPS initiated the statin prescription. Descriptive statistics were used to summarize outreach and statin prescribing data. Prescription drug event data were also collected from the health plan to verify SUPD measure performance.

RESULTS: At the start of 2018, DHMP's performance on the SUPD measure was $65.7 \%$ (Medicare Advantage Part D national average was $68.5 \%$ ). of the 326 patients whose charts were reviewed and who were contacted, $275(84.4 \%)$ were eligible for statin initiation, and of these, $115(41.8 \%)$ were prescribed statin therapy. The increase in statin prescribing and dispensing increased DHMP's performance on the SUPD measure to $87.1 \%$ at the end of 2018, which correlates with a 5-star rating based on the 2019 cut points.

CONCLUSIONS: CPS embedded in the PCMH setting are well positioned to participate in and positively affect population health initiatives such as the SUPD measure. Appropriate prescribing of statin therapy by CPS for patients included in the SUPD measure ensures that they are on key medication therapy for mitigating atherosclerotic cardiovascular disease and may improve a health plan's Medicare star rating.

J Manag Care Spec Pharm. 2020;26(7):910-17

Copyright $\odot 2020$, Academy of Managed Care Pharmacy. All rights reserved.

\section{What is already known about this subject}

Patients with diabetes are at high risk for complications related to atherosclerotic cardiovascular disease (ASCVD).

Use of statin medications in patients with diabetes is key for mitigating development of primary and secondary ASCVD.

The Statin Use in Persons with Diabetes (SUPD) measure is a star rating quality measure by which health plans are graded.

\section{What this study adds}

Population-level management by pharmacists is effective for ensuring that patients with diabetes are receiving appropriate statin therapy.

Pharmacists are well equipped to risk-stratify patients and review their medical histories for statin intolerances, allergies, or contraindications

Direct statin prescribing by pharmacists is an effective way to ensure that patients with diabetes are receiving appropriate statin therapy and to improve SUPD star rating measure metrics

D rimary prevention of cardiovascular disease (CVD) is an important aspect of managing patients with diabetes mellitus (DM). ${ }^{1}$ Many clinical trials have demonstrated the beneficial effect of statins use for primary prevention of CVD. ${ }^{2}$ Statin use in patients with DM is recommended by guidelines from the American Heart Association (AHA)/American College of Cardiology (ACC), American Diabetes Association, American Association of Clinical Endocrinologists/American College of Endocrinology, and National Lipid Association. ${ }^{1,3-7}$ The 2018 AHA/ACC Multisociety Cholesterol Guideline recommends that patients aged $40-75$ years with DM and low-density lipoprotein cholesterol (LDL-C) of $70-189 \mathrm{mg} / \mathrm{dL}$ be started on a moderate-intensity statin. In patients with DM who are at higher risk (e.g., multiple CVD risk factors and aged 50-75 years), high-intensity statins (i.e., atorvastatin $40-80 \mathrm{mg}$ or rosuvastatin $20-40 \mathrm{mg}$ daily) should be used to decrease LDL-C by $\geq 50 \%$. $^{3}$ These are similar recommendations to the previous 2013 ACC/AHA guideline that identified patients with DM as 1 of 4 statin benefit groups and encouraged moderate- or high-intensity statin use in the patients with DM aged 40-75 years cohort. ${ }^{4}$ 
TABLE 1 Statin Use in Persons with Diabetes Measure MAPD Star Cutpoints

\begin{tabular}{l|c|c|c|c|c}
\hline MAPD Year & 1 Star & 2 Stars & 3 Stars & 4 Stars & 5 Stars \\
\hline 2019 & $<72 \%$ & $\geq 72 \%$ to $<76 \%$ & $\geq 76 \%$ to $<80 \%$ & $\geq 80 \%$ to $<83 \%$ & $\geq 83 \%$ \\
\hline
\end{tabular}

$\mathrm{MAPD}=$ Medicare Advantage prescription drug plan

In October 2017, our health system's ambulatory pharmacy patient-centered medical home (PCMH) team, which consists of 7 clinical pharmacy specialists (CPS) embedded within 5 medical homes, identified the Pharmacy Quality Alliance (PQA) and National Quality Forum (NQF) measure D-14: Statin Use in Persons with DM (SUPD) as a key clinical and quality measure that we as CPS could positively influence. ${ }^{8}$ This PQA and NQF measure aligns with the 2018 AHA/ACC Multisociety Cholesterol Guideline in its approach to grade health plans on the percentage of patients with DM aged 40-75 years who are prescribed and fill a statin prescription of any intensity (as measured by prescription drug event [PDE] data). ${ }^{4}$ Although the SUPD measure is based on the older 2013 ACC/AHA guideline, the clinical intent to prevent atherosclerotic cardiovascular disease (ASCVD) in primary prevention patients with DM is consistent with current guidelines. The Centers for Medicare \& Medicaid Services (CMS) added the SUPD measure as a display measure in 2015, and it became an official Part D star measure in 2019 using 2017 data. ${ }^{9}$

CMS operates a Star Rating Program for Medicare Advantage plans, which is a 5-star quality rating system that measures a plan's performance on selected quality measures, such as the SUPD measure (Table 1). ${ }^{10}$ Star ratings for Medicare Advantage prescription drug (MAPD) plans are used to measure the quality of the health plan, to assist beneficiaries in finding and selecting the best plan for them, and to determine Medicare Advantage quality bonus payments. ${ }^{10}$ The ultimate goal of the star ratings is to improve the level of accountability and quality of health care organizations and their providers. ${ }^{9}$

Our health system, Denver Health Medical Center, owns a nonprofit health plan, Denver Health Medical Plan (DHMP), which offers commercial, Medicaid, and Medicare insurance products. Our PCMH team developed a population health intervention that involved collaboration with DHMP to identify and perform interventions with Medicare patients who fit the SUPD criteria: patients who received at least 2 DM medication fills, were aged $40-75$ years, and were not currently prescribed a statin. At the beginning of the star measure reporting period in 2017, DHMP was performing at the MAPD average (70.3\% vs. $69.0 \%$ ). This score ultimately increased to $79 \%$ for 2017 , correlating to a 3-star rating for 2019 (Table 1). At the start of 2018, our SUPD measure performance decreased, and DHMP was performing below the MAPD average (65.7\% vs. 68.5\%), which signaled an opportunity to improve performance.
Performance above the MAPD average is indicative of receipt of a more positive star rating (Table 1).

Our PCMH team, in collaboration with DHMP, created and engaged in this population health initiative related to the SUPD measure as an opportunity to positively affect patient care by ensuring appropriate statin use in patients with indications to statins and, in doing so, increase DHMP's Medicare Advantage star rating. This intervention was also a means to assess the feasibility and potential effect of future CPS-led population health interventions.

Because the SUPD measure has recently became a star measure, there is an urgency among health plans to devote resources to improving or maintaining high performance on the SUPD measure. Little data have been published to describe the optimal way to do this; however, it is widely accepted that CPS are well positioned to positively affect the SUPD measure. Our intervention serves as a means to inform the broader community how a health system and/or health plan may use CPS to improve SUPD measure performance.

\section{Methods}

$\overline{\text { This prospective cohort intervention by CPS on a managed }}$ care population eligible for the SUPD measure occurred within a nonprofit health system that serves an urban, underserved, safety-net population. The intervention was approved by the Denver Health Quality Improvement Review Committee, the Denver Health Sponsored Programs and Research Office, and the Colorado Multiple Institutional Review Board as a quality improvement (QI) project based on the determination that it was nonhuman subjects research.

In January 2018, CPS received a list of health plan patients eligible for SUPD intervention because they were aged 40-75 years and had received at least 2 DM medication fills (e.g., insulin, metformin, and sulfonylurea) within the past year and were not currently prescribed (or not filling a prescribed) a statin of any intensity. Although patients with DM are indicated for at least a statin of moderate intensity, the SUPD measure counts any statin prescription of any intensity as meeting the measure. Patients included in the SUPD list could have been statin naive or have previously received a statin. Those patients with a previous statin history were not actively filling a statin prescription at the time of the project, and the reason for that was unknown until the CPS performed an in-depth chart review.

These patient lists were compiled using data from Acumen, a validated third-party analytics vendor that calculates Medicare 


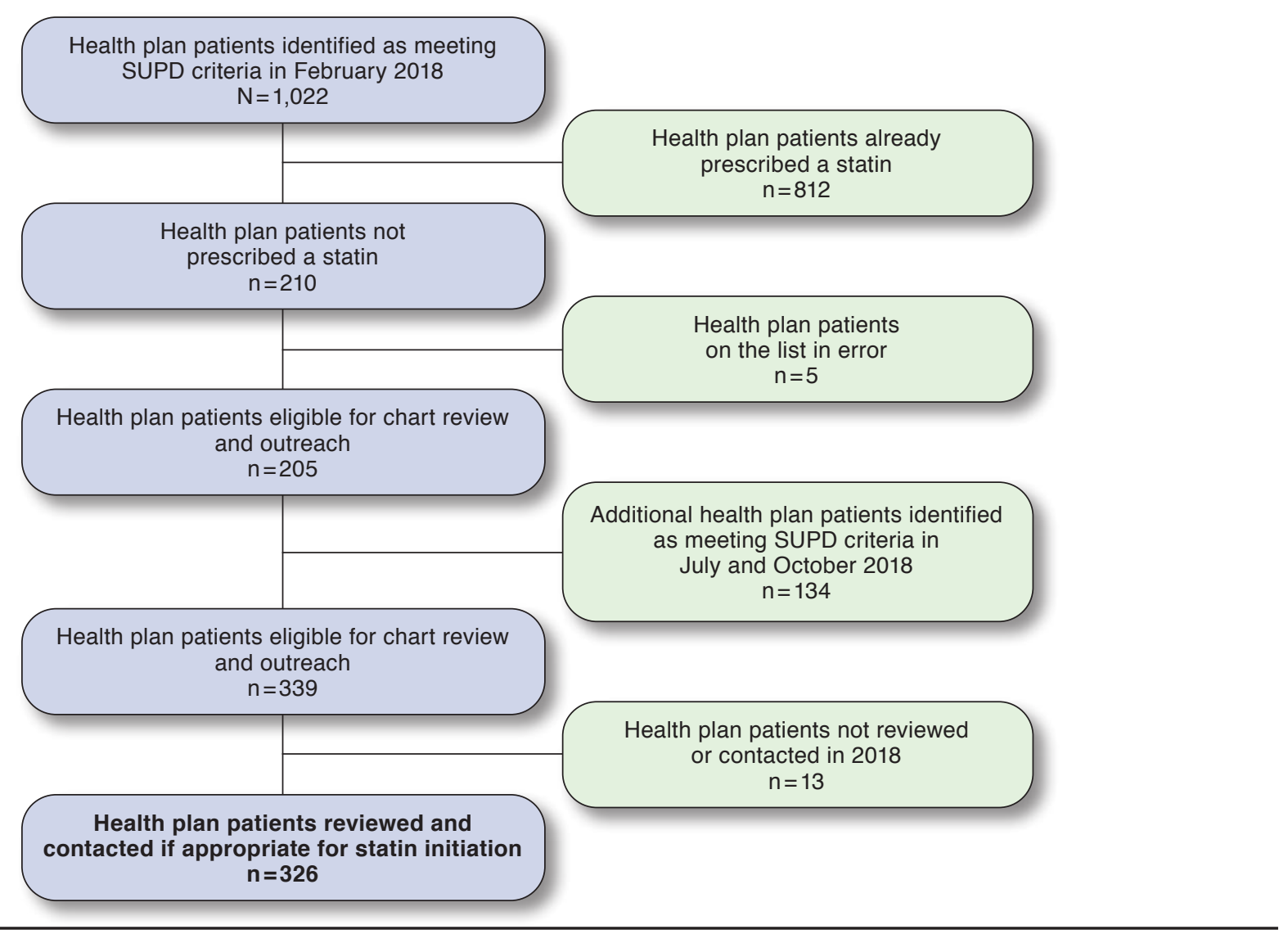

SUPD $=$ Statin Use in Persons with Diabetes measure.

Part D data from DHMP's PDEs. With the patient-level data supplied by Acumen, the DHMP Pharmacy and QI teams then added the necessary data elements that allowed for specific targeting of patients by the PCMHs. The patient list was divided among the PCMH team members so that each CPS received a list of patients within their medical homes. Patients in the remaining 4 medical homes that did not have an embedded CPS were equally distributed among the team so that each CPS had approximately the same number of patients for chart review and outreach.

As a PCMH team, we developed an outreach plan; processes for ordering statin therapy, either according to an existing collaborative drug therapy management (CDTM) protocol or with a provider cosignature; and standardized documentation in Epic (the electronic health record [EHR]; Verona, WI). Statin prescriptions and/or laboratory orders placed per CDTM for patients already referred for CPS management did not require a provider cosignature as specified in our protocol. Statin prescriptions and/or laboratory orders for patients not managed by CDTM were processed nearly identically, with the exception that they required a provider cosignature. The prompt for provider cosignature was the means by which we requested provider acceptance of the intervention; if the order was cosigned then the provider approved of the initiation of a statin prescription for that patient.

We tracked data in Excel (Microsoft, Redmond, WA) spreadsheets on a secure DH server. Patient chart review and outreach began in February 2018 and concluded in December 2018. Subsequent to the list provided to CPS in January 2018, updated lists of SUPD-eligible patients were provided in July and October 2018 to account for newly eligible members.

\section{Results}

\section{Patient Characteristics}

Of the initial 1,022 health plan patients who fit the SUPD criteria, 210 were identified as not having a prescribed statin as of January 2018. After excluding 5 patients who had passed away or were ineligible for the SUPD measure (i.e., diagnosed 


\begin{tabular}{|c|c|c|}
\hline TABLE 2 & $\begin{array}{l}\text { tin Drug and Dose } \\
\text { terns in Health Pla } \\
\text { alifying for the SU }\end{array}$ & $\begin{array}{l}\text { ribing } \\
\text { ents } \\
\text { asure }\end{array}$ \\
\hline Drug & Total Daily Dose (mg) & Count \\
\hline Atorvastatin ${ }^{a}$ & 10,20 & 26 \\
\hline Atorvastatin ${ }^{b}$ & 40,80 & 62 \\
\hline Pravastatinc $^{c}$ & 10,20 & 2 \\
\hline Pravastatin ${ }^{\mathrm{a}}$ & 40,80 & 4 \\
\hline Rosuvastatin $^{\mathrm{a}}$ & 5,10 & 7 \\
\hline Rosuvastatin ${ }^{b}$ & 20,40 & 8 \\
\hline Simvastatinc & 5 & 1 \\
\hline Simvastatin ${ }^{\mathrm{a}}$ & 20,40 & 5 \\
\hline \multicolumn{3}{|c|}{$\begin{array}{l}{ }^{a} \text { Moderate-intensity statin drug and dose. } \\
b \text { High-intensity statin drug and dose. } \\
\text { Low-intensity statin drug and dose. } \\
\text { SUPD = Statin Use in Persons with Diabetes measure. }\end{array}$} \\
\hline
\end{tabular}

with pre-DM instead of DM), we had a list of 205 patients for chart review and outreach. An additional 134 SUPD-eligible patients were identified in July and October 2018, for a total of 339 patients identified during the calendar year 2018 as not meeting the SUPD measure.

Within this patient cohort, the mean (standard deviation [SD]) age was 63 (7.7) years; mean (SD) glycosylated hemoglobin Alc (Alc) was 8.0\% (1.9\%); and mean (SD) atherosclerotic cardiovascular disease (ASCVD) risk score was 21.7\% (14.2\%). These ASCVD data indicated that most patients who were not prescribed a statin warranted therapy with a high-intensity statin based on the 2013 ACC/AHA cholesterol guidelines, which recommended high-intensity statin therapy for an ASCVD risk score $\geq 7.5 \%$. The 2018 AHA/ACC cholesterol guidelines recommends moderate-intensity statins for anyone with DM regardless of LDL-C and/or ASCVD risk score. ${ }^{3}$

\section{Pharmacist Intervention}

Of the 339 health plan patients identified as having DM who were not currently prescribed or filling their prescribed statin therapy, 326 had their charts reviewed by a CPS and were contacted if statin therapy was deemed appropriate in 2018 (Figure 1). A total of 275 patients (84.4\%) were eligible for statin therapy (i.e., no contraindication existed), and of these, 115 patients were newly prescribed or represcribed statin therapy, a success rate of $41.8 \%$. A majority of these patients $(n=84,73.0 \%)$ who were prescribed a statin were empaneled in PCMH clinics that had a CPS; however, there was no difference in statin prescribing success related to whether there was a CPS in the patient's medical home or not (84 vs. $31 ; P=0.629$ ).

As previously mentioned, a majority of patients identified as needing statin therapy warranted high-intensity statin therapy based on the mean ASCVD risk score and the 2013 ACC/AHA cholesterol guideline that informed the SUPD measure.
A breakdown of statin drugs and doses prescribed are included in Table 2. Of the 115 statin prescriptions ordered during the project time period, 70 (60.9\%) were for a highintensity statin (atorvastatin $40 \mathrm{mg}$ to $80 \mathrm{mg}$ or rosuvastatin $20 \mathrm{mg}$ to $40 \mathrm{mg}$ daily). All patients ( $\mathrm{n}=115,100 \%)$ were prescribed an appropriate intensity of statin, given their clinical circumstances. Nearly all patients ( $\mathrm{n}=112,97.4 \%$ ) were prescribed either a moderate- or high-intensity statin, which fits the SUPD measure and the recommendations for statin intensity for patients with diabetes in the updated 2018 AHA/ ACC Multisociety Cholesterol Guideline. ${ }^{3}$ The 3 patients (2.6\%) who were prescribed a low-intensity statin had tolerability issues that precluded use of a moderate- or high-intensity statin. Based on the structure of the intervention, the CPS did not directly follow-up on statin adherence. However, the CPS prescribed a 90-day supply with 3 refills in order to maximize patients' access to their statins.

Within the population of 326 patients whose charts were reviewed and/or who were contacted, there were 43 patients (13.2\%) who had a contraindication to statin therapy, and 8 patients $(2.5 \%)$ with a documented statin intolerance or allergy. Of the remaining patients, 64 (23.3\%) refused statin initiation or reinitiation; 6 patient providers refused statin initiation (2.2\%); 41 patients were unreachable by phone despite 3 phone call attempts (14.9\%); and 49 patients (17.1\%) had another reason for not starting a statin (e.g., patient was out of the country at the time of the call). Reasons for statin declination were not systematically captured for each patient, but anecdotal patient responses for declining a statin prescription frequently included concerns for added cost and/ or pill burden and not believing statin therapy was necessary. The most common reason for provider refusal was that the provider wanted to see the patient for an appointment and discuss in person.

\section{Health Plan Star Measure Improvements}

Our 2018 report from CMS demonstrated that our health plan started the 2018 benefits year with $65.7 \%$ of health plan members in the SUPD cohort having prescription claims data for statin therapy, which was below the MAPD average. The final report that the health plan received for 2018 indicated that this proportion had increased to $87.1 \%$ (Figure 2). Our health plan's improvements are exceeding that of the national average of other MAPD plans (improvement from $68.5 \%$ to $81.4 \%$ over the same period), and our measured improvements are more pronounced than that of the national average. Our improvement in the SUPD measure represents an absolute increase of $21.4 \%$ and a relative increase of $32.6 \%$ compared with an absolute increase of $12.9 \%$ and a relative increase of $18.8 \%$ across the average of all other MAPDs. Based on the 2019 star rating cut points ( $\geq 83 \%$ indicates a 5 -star rating), we can reasonably anticipate that the 2018 SUPD data will 


\section{FIGURE 2 MAPD Statin Use in Persons with Diabetes Measure Performance, 2018}

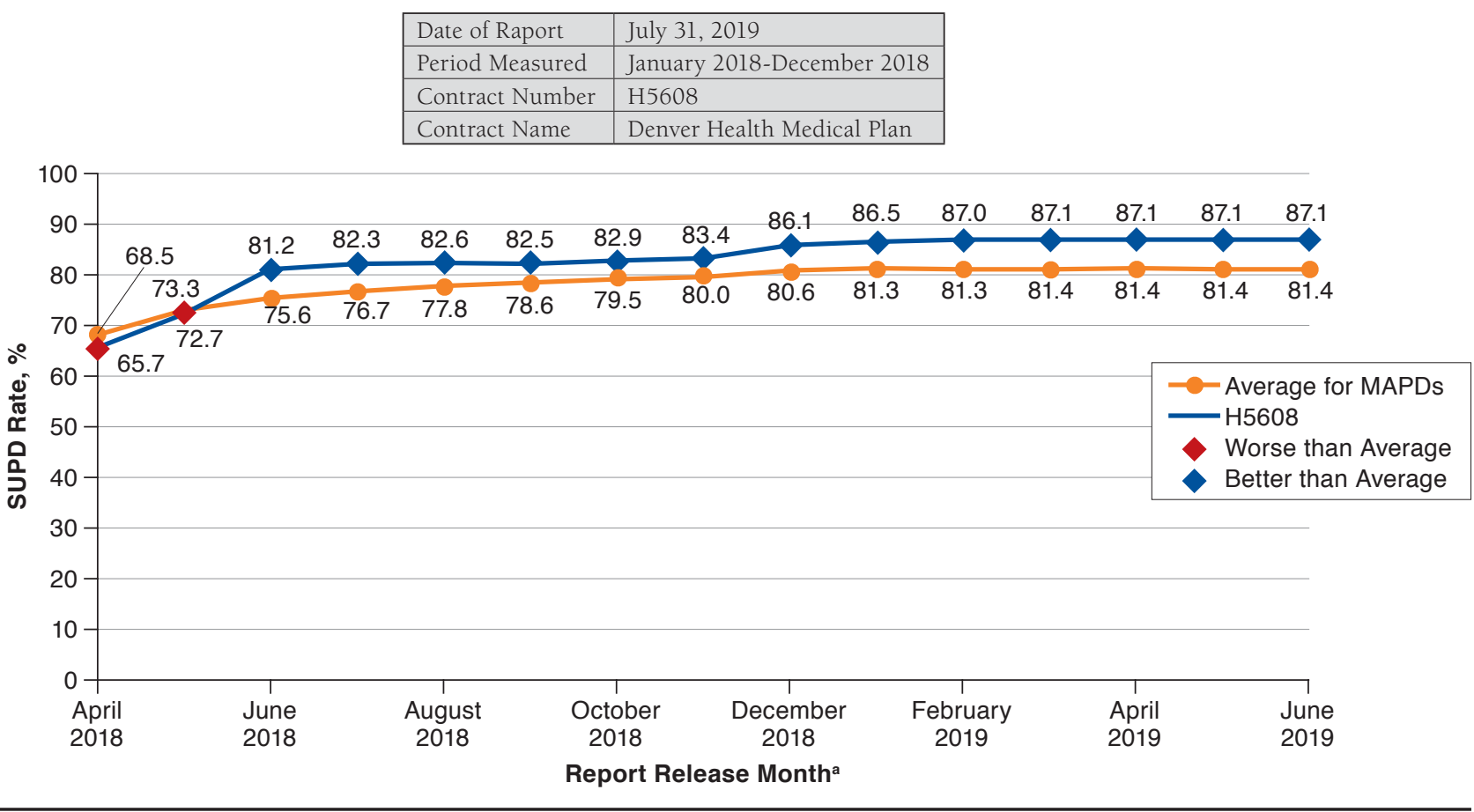

Note: This chart compares the contract's monthly SUPD rate to average rate for the contract type. Red data points highlight the months the contract performed worse than the average.

aThe SUPD rate contains PDE data with 2018 dates that were submitted up to 1 month before the report release date. Therefore, the SUPD rate of a report released in April 2018 contains PDE data with dates of service from January 2018 through March 2018 that were submitted by March 2018.

$M A P D=$ Medicare Advantage Prescription Drug plan; PDE= prescription drug event; SUPD =Statin Use in Persons with Diabetes.

correlate to a 5-star rating for the SUPD measure in 2020 for our health plan.

\section{Discussion}

$\overline{\mathrm{CPS}}$ are well positioned to affect population health efforts. ${ }^{11} \mathrm{An}$ editorial by Sanborn (2017) describes the role of the pharmacist, including those embedded in a PCMH, in addressing population health initiatives to better deliver comprehensive patient care. ${ }^{11}$ Sanborn explains the importance of developing metrics to measure patient outcomes and to strategically align these with the health system's priorities and quality outcomes initiatives with a goal of justifying additional services or resources. ${ }^{11}$ Our partnership with DHMP on this SUPD intervention allowed us to align our work with a systemwide strategic initiative to potentially improve DHMP's overall star rating and to further quantify the value of PCMH-based CPS positions.

An editorial by Shermock (2017) specifically references CMS and its 5-star quality rating system and how pharmacists can positively affect the incentives offered by improving medication adherence for select chronic diseases states and providing point-of-service interventions for DM and high-risk medications. ${ }^{12}$ We have demonstrated through our SUPD intervention the ability to identify and partner with DHMP to improve patient care and for the opportunity to positively affect its star rating. Our health plan's SUPD star rating for 2019 (based on 2017 data, preintervention) was a 3 based on 79\% measure performance. We are confident that our 2020 SUPD star rating measure performance will reflect positively on our ability to initiate statin therapy in our health plan patients with DM as it aligns with a 5-star rating. In addition, the successes of this project set the foundation for the ability of CPS within the PCMH to improve other Part D star measures through direct, targeted interventions. Our data were used to support the addition of a 0.4 full-time equivalent of clinical pharmacist time for population health interventions, which was hired in the fall of 2019.

While there are many examples of pharmacist involvement in the care of patients with DM, there is a paucity of literature describing the pharmacist's role in DM population health initiatives. However, a study by Rana et al. (2018) highlighted the ability of pharmacists working under protocols to assist in a DM-related population health initiative known as "PHASE" 
(Preventing Heart Attacks and Strokes Every Day). ${ }^{13}$ The PHASE program consisted of a 4-pronged intervention to improve glycemic control, lipids, and blood pressure among Kaiser Permanente Northern California (KPNC) patients with DM. One of the interventions included pharmacist care managers who were referred patients by the primary care provider and identified, contacted, educated, and treated eligible patients with DM whose cardiovascular risk factors could be managed per protocol. Specifically looking at statin use and lipid control, pharmacists served as care managers to titrate medications (e.g., statins) per protocol and were key in assisting in this intervention that increased LDL-C control (defined as LDL-C $<100 \mathrm{mg} / \mathrm{dL})$ from $47 \%$ to $71 \%(P<0.05)$ over the 10 -year study period from 2004-2013, compared with no significant improvement nationally based on Healthcare Effectiveness Data and Information Set data (40\%-44\%; P = 0.20). Rana et al. concluded that there was substantially greater improvement in cardiovascular risk factor control in KPNC patients with DM after implementation of a population management program that used pharmacists as a cornerstone of management. ${ }^{13}$

In a recently published retrospective cohort analysis, Troksa et al. (2020) evaluated the rate of provider statin prescribing following CPS statin recommendations for patients with type 2 $\mathrm{DM}$ and an indication for statin therapy. ${ }^{14}$ In this intervention, centrally located and embedded CPS received a list of patients with type $2 \mathrm{DM}$ who were not prescribed a statin. Both types of CPS reviewed these patients' charts and then made recommendations to providers regarding statin initiation using 2 approaches: (1) upcoming visit approach (targeted patients with a provider appointment in the next 7 days) and (2) prospective patient panel approach (a priori chart review by a centrally located CPS of patients without an upcoming appointment). The single strategy cohort included only the upcoming visit approach and the dual strategy cohort included both approaches. In total, CPS made statin recommendations for 793 patients. However, based on time constraints, only 400 patients (200 from each cohort) were randomly selected for evaluation.

The statin initiation rate using the upcoming visit approach in each cohort was similar (46\% and 38.7\%; $P=0.25$ ) and was lower with the prospective panel approach (31.5\%; $P=0.049$, compared with combined data for the upcoming visit approach). Troksa et al. commented that, since the start of the population health initiative, statin prescribing rates across all eligible patients with type 2 DM increased from $69.3 \%$ to $74.7 \%$. The authors concluded that CPS are effective at recommending statin initiation in eligible patients, and using the upcoming visit approach led to higher rates of prescriberbased statin initiation. ${ }^{14}$

Vanderholm et al. (2018) published an opinion piece on pharmacist involvement in SUPD, which suggested that pharmacist prescriptive authority for statin prescribing is a means to close the gap on patients with diabetes who are eligible for but not yet prescribed a statin. ${ }^{15}$ This is a different approach than what was taken in the previously published studies (pharmacist-led statin titration and pharmacist-led statin initiation recommendations made to providers) and similar to our SUPD intervention. Vanderholm et al. proposed that pharmacists are able to collect all relevant information, including liver function tests, and make patient-specific pharmacotherapeutic plans that includes follow-up and monitoring. The goal of using pharmacist prescriptive authority to initiate statin therapy in patients with DM could lead to earlier statin initiation and reduce inconveniences associated with having to communicate with patient providers in order to get statins prescribed. ${ }^{15}$ With our SUPD intervention, we were able to demonstrate the ability to initiate a prescription for statin therapy where it was omitted, as well as reinitiate statin therapy and encourage adherence in patients who had been prescribed a statin but had stopped filling it.

We identified several barriers to CPS-led statin prescribing, including patient misperception or misunderstanding of statin importance, cost and pill burden concerns, and providers requesting ownership of the decision-making process. Patient misperceptions and misunderstandings may be improved through education, which may require a face-to-face visit or provision of a patient-friendly educational handout. Cost and pill burden concerns could be alleviated with a comprehensive medication management review that could evaluate opportunities for deprescribing or prescribing of combination medications when appropriate. Provider barriers could be clarified by surveying provider groups regarding their concerns or questions about statin prescribing and then improved by developing targeted educational approaches, depending on the outcomes of the surveys.

\section{Limitations}

Our intervention project for the SUPD measure has several limitations that should be noted. First, this project was not designed to ascertain causality, so we cannot conclude that the intervention performed by CPS is the only mechanism that increased SUPD measure performance. While we recognize that the direct statin prescribing by CPS for patients in need may not account for the entire increase in SUPD measure performance, we postulate that the education of providers about our initiative, as well as our encounters documented in the EHR as we reviewed charts of patients eligible for the SUPD intervention, passively educated and prompted many providers to consider statin prescribing for their own patients. These may have been confounders that positively contributed to the overall improvement in DHMP's SUPD measure performance.

Second, determination of statin contraindication, intolerance, and allergy was achieved through manual chart review by the CPS. If information regarding any of these 
issues was incorrectly documented in a patient's EHR, a viable statin candidate may have been bypassed for outreach. The Acumen reports used for patient-level detail also had a data lag of several months to allow for claims and PDE run-out. Ongoing technical refinements in data extraction from health plan claims, PDEs, and EHRs will help improve our ability to mine administrative data and supplement the EHR review process. Related to this limitation, it is unknown if the patients noted to have a statin intolerance were rechallenged and were still unable to tolerate a statin; these may be patients where an opportunity to start a statin was missed. Better identification of statin intolerances, allergies, and drug-drug interactions by the CPS could create a more robust intervention in the future.

Third, despite most patients requiring a high-intensity statin based on the 2013 ACC/AHA cholesterol guidelines (on which the SUPD measure is based), only $61 \%$ of these patients were initiated on a high-intensity statin. The SUPD measure does not grade a health plan based on statin intensity; however, it is clinically important to have high-risk patients on the appropriate statin intensity for the best clinical outcome.

Fourth, there is no available Acumen report of LDL-C levels achieved after statin initiation. The PQA SUPD measure is classified as a process measure, since prescribing a statin is noted to be a "step that should be followed to provide good care" rather than an outcome of such care. Process measures such as the SUPD focus on whether actions that have been shown to benefit patients have been followed, but they do not require a report on clinical outcomes achieved (e.g., LDL-C levels). ${ }^{8}$

Fifth, there were a few patients who had drug-drug interactions (e.g., concurrent use of fenofibrate) or statin intolerance issues that necessitated prescription of a lower potency statin than indicated by the ASCVD risk scores; however, this does not account for the majority of those patints who may have been prescribed a moderate- or low-intensity statin. According to the 2018 AHA/ACC Multisociety Cholesterol Guideline update, there is no longer a recommendation to calculate an ASCVD risk score for patients with DM in order to determine statin potency, but rather, age and major ASCVD risk factors should be considered for escalating statin intensity. ${ }^{3}$ We did not have ASCVD risk factor information at the time this project was implemented so were unable to speculate on how many patients would be indicated for high-intensity statins based on the updated 2018 guidelines. We also were unable to capture patients with DM who were prescribed and filling a low-intensity statin; this could have been a patient population to positively affect as well, since guideline recommendations suggest patients with DM should be prescribed at least a moderate-intensity statin. ${ }^{3}$

Sixth, we were unable to capture adherence after statin initiation beyond the sustained increase in SUPD measure performance. It is possible that a patient could have filled a statin prescription 1 time during the year and been counted as meeting the measure, despite not refilling it after the first fill. Similarly, a patient could have been filling a statin prescription but not taking it, which could have been reflected in LDL-C measurements. A future iteration of this intervention should include ongoing assessment of patient adherence and tolerability beyond the first fill. We are, however, encouraged by our ongoing DHMP SUPD reports that continue to demonstrate sustained adherence to the SUPD measure of $>87 \%$.

Finally, this intervention project was conducted at 1 health system in the Denver metro area. The patient population in general represented an urban, diverse, and underserved population. While our CPS-led statin prescribing intervention can likely be applied in many other health systems, the details, successes, and challenges will likely vary by geographic region and patient population.

To our knowledge, our project and intervention is the first in which a PCMH team of CPS partnered with their health system's health plan to design and implement a population health initiative aimed at improving patient care and the plan's Medicare Advantage star rating related to the new SUPD measure by directly prescribing statin therapy to eligible patients. Our intervention succeeded in increasing statin use in a high-risk population and also increased the rate of DHMP members who were prescribed statin therapy, thereby increasing our health plan's performance on the SUPD measure.

Anecdotally, we have continued to see a trickledown effect of increased statin prescribing in SUPD-eligible patients as providers have reviewed our SUPD encounters in the EHR and incorporated it in their practices. Our project's effect on DHMP has potential positive financial implications that will be realized in the future through an improved overall Medicare plan star rating. While we cannot project actual financial gains that will be realized in 2020 with the increase in the star rating, achievement of a 5-star rating is associated with increased Medicare payments per member per month, bonus payments, and additional rebates that will allow the plan to provide more value to our members. ${ }^{16}$ This intervention also serves as a basis for ongoing CPS-led collaborative initiatives within our health system and sets an example for how to implement and succeed with such an intervention in other health systems.

\section{Conclusions}

CPS in the ambulatory care PCMH setting are well positioned to participate in and positively affect population health initiatives such as the SUPD measure. Appropriately prescribing or represcribing statin therapy by CPS for patients with DM aged 40-75 years ensures that patients are on key medication therapy for mitigating ASCVD risk. The financial benefits that will be realized by our health system with the improvement of the Medicare star rating related to the SUPD measure justify 
the expense of using CPS resources for this type of population health initiative. Bonus payments are given to plans that achieve a total overall plan star rating of 4 or higher. High Medicare star ratings also serve as a roadmap for functional health plan operation. Our CPS-led intervention and its outcomes serve as a model for other institutions around the country that are actively engaged in improving appropriate statin use in patients with DM.

\section{Authors}

SARAH L. ANDERSON, PharmD, and JOEL C. MARRS, PharmD, MPH, University of Colorado Skaggs School of Pharmacy and Pharmaceutical Sciences, Aurora. CYNTHIA R. CHACHAS, PharmD, Denver Health Medical Plan, Denver, Colorado; BRIAN S. CICHON, MPH, Colorado Access, Aurora; AMBER D. CIZMIC, PharmD, Denver Health Medical Center, Denver, Colorado; BIANCA B. CALDERON, PharmD, Regis University School of Pharmacy, Denver, Colorado; and TARA B. VLASIMSKY, PharmD, Ambulatory Clinical Pharmacy, SCL Health, Broomfield, Colorado.

AUTHOR CORRESPONDENCE: Sarah L. Anderson, PharmD, Associate Professor, University of Colorado Skaggs School of Pharmacy and Pharmaceutical Sciences, 12850 E. Montview Blvd., Mail Stop C238, Aurora, CO 80045. Tel.: 303.724.5926;

E-mail: Sarah.Anderson@cuanschutz.edu.

\section{DISCLOSURES}

This was an unfunded, investigator-initiated project. Anderson owns stock in Merck \& Co. All other authors have no conflicts of interest to disclose.

\section{ACKNOWLEDGMENTS}

The authors thanks Adrian Boka, PharmD; Paul Cornelison, PharmD; Josh Gannon, PharmD; Julianna Kluemper, PharmD; Leticia Smith, PharmD; and Brooke Wobeter, PharmD, for their participation in the intervention and data collection.

\section{REFERENCES}

1. American Diabetes Association. 10. Cardiovascular disease and risk management: Standards of Medical Care in Diabetes-2020. Diabetes Care. 2020;43(Suppl 1):S111-S134.

2. Collins R, Reith C, Emberson J, et al. Interpretation of the evidence for the efficacy and safety of statin therapy. Lancet. 2016;388(10059):2532-61.
3. Grundy SM, Stone NJ, Bailey AL, et al. 2018 AHA/ACC/AACVPR/AAPA/ ABC/ACPM/AGS/APhA/ASPC/NLA/PCNA guideline on the management of blood cholesterol: a report of the American College of Cardiology/American Heart Association Task Force on Clinical Practice Guidelines. J Am Coll Cardiol. 2019;73(24):e285-e350.

4. Stone NJ, Robinson JG, Lichetenstein AH, et al. 2013 ACC/AHA guideline on the treatment of blood cholesterol to reduce atherosclerotic cardiovascular risk in adults: a report of the American College of Cardiology/American Heart Association Task Force on Practice Guidelines. J Am Coll Cardiol. 2014;63(25 Pt B):2889-934.

5. Jacobson TA, Ito MK, Maki KC, et al. National Lipid Association recommendations for patient-centered management of dyslipidemia: part 1-full report. J Clin Lipidol. 2015;9(2):129-69.

6. Garber AJ, Abrahamson MJ, Barzilay JI, et al. Consensus statement by the American Association of Clinical Endocrinologists and American College of Endocrinology on the comprehensive type 2 diabetes management algorithm-2019 executive summary. Endocr Pract. 2019;25(1):69-100.

7. Jacobson TA, Maki KC, Orringer CE, et al. National Lipid Association recommendations for patient-centered management of dyslipidemia: part 2 J Clin Lipidol. 2015;9(6):S1-S122.

8. Pharmacy Quality Alliance. Statin use in persons with diabetes (SUPD). October 2019. Available at: https://www.pqaalliance.org/measuresoverview\#supd. Accessed May 22, 2020.

9. Centers for Medicare \& Medicaid Services. 2017 star ratings. October 2016. Available at: https://www.cms.gov/newsroom/fact-sheets/2017-starratings. Accessed May 22, 2020.

10. Medicare Payment Advisory Commission. Chapter 8: Redesigning the Medicare Advantage quality bonus program. In: Report to the Congress: Medicare and the Health Care Delivery System. June 2019. Available at: http://www.medpac.gov/docs/default-source/reports/jun19_ch8_medpac_ reporttocongress_sec.pdf. Accessed May 22, 2020.

11. Sanborn MD. Population health management and the pharmacist's role. Am J Health Syst Pharm. 2017;74(18):1400-01.

12. Shermock KM. Population health management: challenges and opportunities for pharmacy. Am J Health Syst Pharm. 2017;74(18):1398-99.

13. Rana JS, Karter AJ, Liu JY, Moffet HH, Jaffe MG. Improved cardiovascular risk factors control associated with a large-scale population management program among diabetes patients. Am J Med. 2018;131(6):661-68.

14. Troksa KA, Billups SJ, Claus LW, Vande Griend JP, Saseen JJ. Effectiveness of a pharmacist-led population health approach to implementing statin therapy in primary prevention patients with type 2 diabetes mellitus. J Am Coll Clin Pharm. February 24, 2020 [Epub ahead of print]. Available at: https://accpjournals.onlinelibrary.wiley.com/doi/full/10.1002/jac5.1222. Accessed May 22, 2020.

15. Vanderholm T, Renner HM, Stolpe SF, Adams AJ. An innovative approach to improving the proposed CMS star rating "statin use in persons with diabetes." J Manag Care Spec Pharm. 2018;24(11):1126-29. Available at: https://www.jmcp.org/doi/10.18553/jmcp.2018.24.11.1126.

16. Bajner R, Meinkow E, Munroe J, Smith JR, McHugh J. Impact of star ratings on Medicare advantage plan success. Becker's Hospital Review. March 15, 2018. Available at: https://www.beckershospitalreview.com/ finance/impact-of-star-ratings-on-medicare-advantage-plan-success.html. Accessed May 22, 2020. 